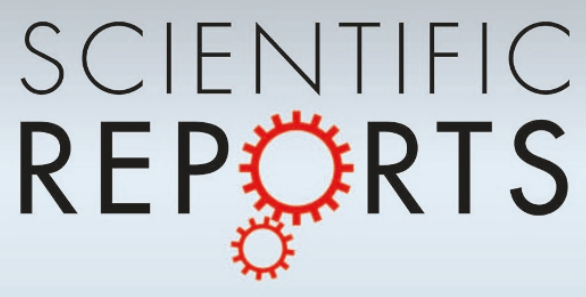

OPEN

SUBJECT AREAS:

PRECLINICAL RESEARCH

RADIONUCLIDE IMAGING

MYOCARDIAL INFARCTION

CELL DEATH

Received

7 August 2013

Accepted

29 September 2014

Published

29 October 2014

Correspondence and requests for materials should be addressed to

A.P. (artiom.petrov@ mountsinai.org) or J.N. (narula@mountsinai.

org)

* These authors contributed equally to this work.

\section{Noninvasive Molecular Imaging of Cell Death in Myocardial Infarction using ${ }^{11} 1 \mathrm{In}-\mathrm{GSAO}$}

Nobuhiro Tahara ${ }^{1 *}$, H. Reinier Zandbergen ${ }^{2 *}$, Hans J. de Haas ${ }^{3,4 *}$, Artiom Petrov ${ }^{3}$, Raghu Pandurangi ${ }^{5}$, Takayoshi Yamaki ${ }^{6}$, Jun Zhou ${ }^{3}$, Tsutomu Imaizumi' ${ }^{1}$, Riemer H. J. A. Slart ${ }^{4}$, Mary Dyszlewski ${ }^{5}$, Tiziano Scarabelli ${ }^{3}$, Annapoorna Kini ${ }^{3}$, Chris Reutelingsperger ${ }^{2}$, Navneet Narula ${ }^{7}$, Valentin Fuster ${ }^{3,8}$ \& Jagat Narula $^{3}$

'Division of Cardio-Vascular Medicine, Department of Medicine, Kurume University School of Medicine, Kurume, Japan, ${ }^{2}$ Cardiovascular Research Institute Maastricht, Maastricht University, Maastricht, Netherlands, ${ }^{3}$ Zena and Michael A. Wiener Cardiovascular Institute, Icahn School of Medicine at Mount Sinai, New York, New York, ${ }^{4}$ Department of Nuclear Medicine and Molecular Imaging, University of Groningen, University Medical Center Groningen, Groningen, the Netherlands, ${ }^{5}$ Covidien Imaging Solutions, Hazelwood, Missouri, ${ }^{6}$ Department of Cardiology and Hematology, Fukushima Medical University, Fukushima, Japan, ${ }^{7}$ Department of Pathology, Weill Cornell Medical College, New York, NY, ${ }^{8}$ Centro Nacional de Investigaciones Cardiovasculares (CNIC), Madrid, Spain.

Acute insult to the myocardium is associated with substantial loss of cardiomyocytes during the process of myocardial infarction. In this setting, apoptosis (programmed cell death) and necrosis may operate on a continuum. Because the latter is characterized by the loss of sarcolemmal integrity, we propose that an appropriately labeled tracer directed at a ubiquitously present intracellular moiety would allow non-invasive definition of cardiomyocyte necrosis. A trivalent arsenic peptide, GSAO (4-(N-(S-glutathionylacetyl)amino)phenylarsonous acid), is capable of binding to intracellular dithiol molecules such as HSP90 and filamin-A. Since GSAO is membrane impermeable and dithiol molecules abundantly present intracellularly, we propose that myocardial localization would represent sarcolemmal disruption or necrotic cell death. In rabbit and mouse models of myocardial infarction and post-infarct heart failure, we employed In-111-labelled GSAO for noninvasive radionuclide molecular imaging.

${ }^{111} \mathrm{In}$-GSAO uptake was observed within the regions of apoptosis seeking agent- ${ }^{{ }^{99 m} \mathrm{Tc}-A n n e x i n}$ A5 uptake, suggesting the colocalization of apoptotic and necrotic cell death processes.

ell death plays a central role in various cardiovascular diseases. Two morphologically distinct modes of cell death - apoptosis (a programmed process characterized by enzymatic degradation and clean removal of the cell) and necrosis (an uncontrolled process characterized by cell swelling, membrane rupture and spill of its contents) have been reported to contribute to the myocardial tissue loss. It is being increasingly realized that apoptosis and necrosis, rather than being entirely independent, may operate on a continuum, at least in response to noxious stimuli ${ }^{1-3}$.

Numerous strategies have been proposed for the detection of cell death early after onset of ischemia within the time window amenable to intervention. Most experience for the recognition of apoptosis has been obtained with single photon emission computed tomography (SPECT) imaging using ${ }^{99 \mathrm{~m}} \mathrm{Tc}$-Annexin A5 (AA5). ${ }^{99 \mathrm{~m}} \mathrm{Tc}$-AA5 targets externalized phosphatidylserine (PS) on membranes of cells with active apoptotic signaling ${ }^{4}$ and the clinical feasibility of ${ }^{99 \mathrm{~m}}$ Tc-AA5 imaging has been demonstrated in the setting of myocardial infarction ${ }^{5,6}$, transplant rejection ${ }^{7}$ and heart failure ${ }^{8}$. On the other hand, several radiotracers targeting necrotic cells through membrane disruption have been developed ${ }^{9-11}$. Notably, antimyosin antibody imaging has been successfully employed for the detection of myocardial necrosis associated with myocardial infarction (MI) ${ }^{9}$, myocarditis ${ }^{9}$, heart failure ${ }^{12}$ and cardiac allograft rejection ${ }^{12}$. However due to technical disadvantages, the necrosis-avid radiotracers have not become popular.

4-(N-(S-glutathionylacetyl)amino)phenylarsonous acid (GSAO) labelled with fluorophores and radionuclides has been used for targeting of cell death in culture, in tumor-bearing mice, and in mice with experimental brain trauma $^{13-15}$. The principle behind GSAO cell death targeting is displayed in figure 1. GSAO is a tripeptide with a 
a

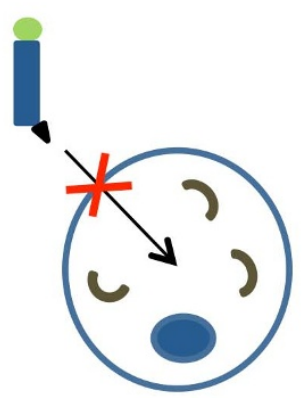

Intact GSAO is membrane impermeable

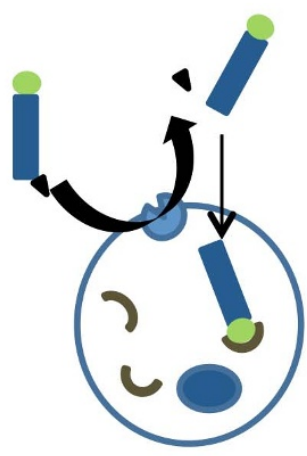

Membrane bound GGT (on proliferating and stressed cells) splices off glutamyl residue and allows cell entrance of metabolite GCAO
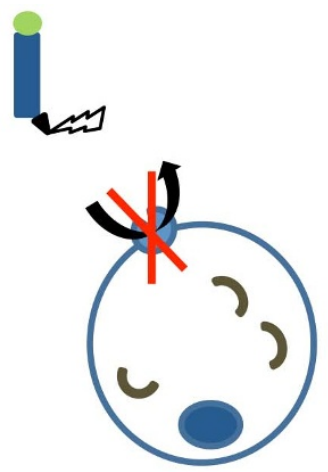

Binding of fluorescent or radioactive reporter molecules to GSAO prevents binding to GGT and thereby prevents all access over intact membrane b

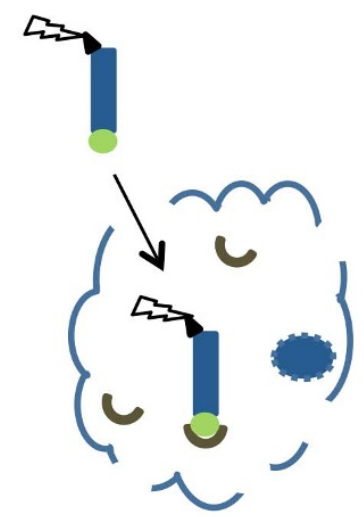

Membrane disruption in necrotic cells allows cell entrance of fluorescently or radiolabeled GSAO and binding to intracellular dithiol molecules

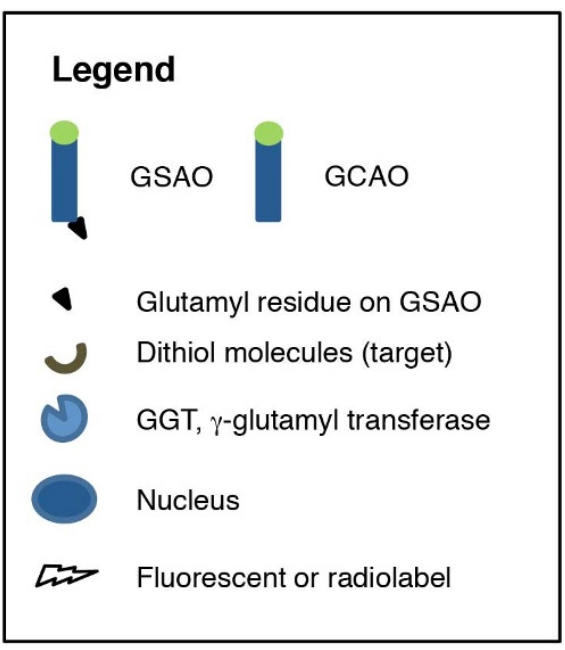

Figure 1 Targeting mechanism of GSAO. GSAO binds to dithiol molecules, which are abundant in the intracellular space and virtually absent in the extracellular space. When labelled with radioactive or fluorescent reporter molecules, GSAO cannot negotiate across the cell membrane of healthy cells (a) but gains free access through disrupted membranes of dying cells (b). Thus, GSAO accumulates in dying cells.

trivalent arsenic moiety. The arsenic group binds to dithiols, which are abundantly present in intracellular milieu and virtually absent from the extracellular space ${ }^{16}$. GSAO cannot reach its intracellular target molecules in intact cells, because it is not able to negotiate across the cell membrane ${ }^{17}$. Cell membrane $\gamma$-glutamyl transferase (GGT) is upregulated during ischemic stress and other situations, splices off GSAO's glutamyl residue and allows cellular entrance of GSAO's metabolite GCAO (4-(N-(S-cysteinylglycylacetyl)amino) phenylarsonous acid $)^{17}$. However, when radioactive or fluorescent reporter molecules are attached to glutamyl residue of GSAO it is not able to bind to GGT, rendering the molecule membrane impermeable ${ }^{13}$. After the necrotic process sets in and the sarcolemmal integritiy is lost; and GSAO gains free entry to the intracellular microenvironment. Of numerous dithiol-bearing intracellular targets including filamin A, eukaryotic translation elongation factor 2, and protein disulfide isomerase (PDI), HSP90 is most widely present ${ }^{13}$. HSP90 comprises approximately $2 \%$ of the intracellular protein content and increases by two-threefold in response to acute stress including ischemia ${ }^{18}$.

In this study we evaluated feasibility of cell death imaging using ${ }^{111}$ In-GSAO in mouse and rabbit models of acute MI and a mouse model of chronic MI. In addition, a subgroup of animals, serial SPECT/CT imaging using ${ }^{99 \mathrm{~m}} \mathrm{Tc}-\mathrm{AA} 5$ and ${ }^{111} \mathrm{In}-\mathrm{GSAO}$ was performed to determine the relationship between these two modes of cell death in the setting of myocardial ischemia and reperfusion. Fluorescent GSAO and AA5 were employed in another subgroup of animals for pathological characterization of the mode of cell death. In addition, ${ }^{111}$ In-GSCA (4-(N-(S-glutathionylacetyl)amino)benzoic acid), which is identical to ${ }^{111}$ In-GSAO except for replacement of the arsenic group with a carboxylic acid group, was used as a negative control compound in radionuclide experiments.

\section{Results}

Fluorescence microscopic characterization of GSAO uptake. Uptake characteristics of GSAO were evaluated and compared with AA5 in mice with acute $\mathrm{MI}$ and sham-operated mice. Experimental acute MI was induced by 30-minute coronary ligation followed by 30 minutes of reperfusion. Sham procedure was identical, except that coronary ligation was not performed. Mice with acute MI $(n=3)$ and sham-operated mice $(n=3)$ were injected Cy5.5-labelled GSAO and Oregon Green-labeled AA5. Qualitative fluorescent microscopic analysis of heart sections showed that cardiomyocytes of sham-operated mice were positive neither for GSAO nor for AA5. In contrast, in ischemia/reperfusioninjured hearts, cardiomyocytes positive for GSAO and AA5 were observed (Fig. 2a-c). Interestingly, all GSAO-positive 

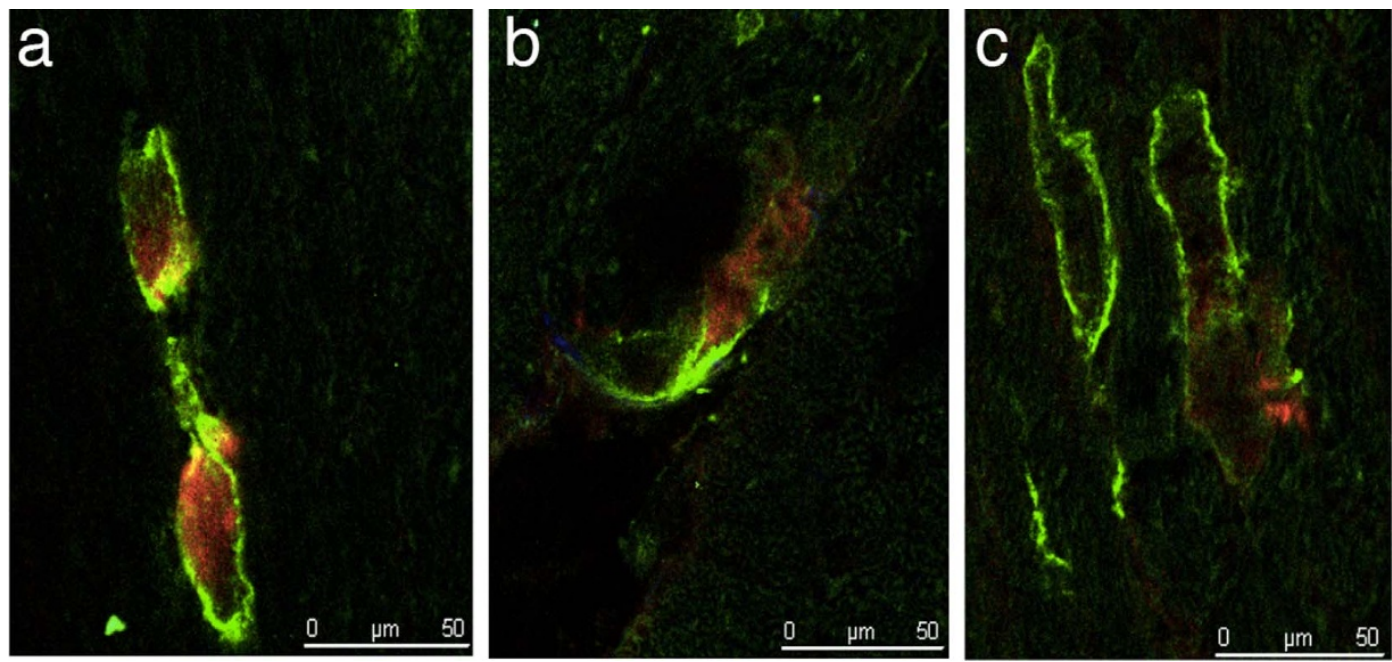

Figure $2 \mid$ Characterization of target binding of GSAO. Tissue sections of acute MI mice, injected fluorescently labeled GSAO and AA5. (a, b) GSAO accumulation (red) was only observed in cells with AA5-positive cell membranes (green). (c) All cells AA5-positive cells were not GSAO positive.

cardiomyocytes bound AA5 (Fig. 2a-c), suggesting that necrosis in the reperfused myocardium coexists with apoptotic signaling. Not all AA5 positive cardiomyocytes had taken up GSAO (Fig. 2c).

Radiolabelled GSAO imaging in acute myocardial infarction. Acute MI was induced in rabbits and mice by coronary ligation for 40 and 30 minutes, respectively. Reperfusion was achieved by removal of the suture and ${ }^{111}$ In-GSAO was administered thirty minutes later. Three hours thereafter, in vivo micro-SPECT/micro$\mathrm{CT}$ imaging was performed, animals were sacrificed and hearts were explanted. Next, ex vivo SPECT/CT and planar cardiac imaging were performed. Then, rabbit hearts were cut into $\sim 32$ small pieces and mice hearts were sectioned in three short axis slices: basal (remote area), middle (border zone) and apical (infarct). Radiotracer uptake was quantified by $\gamma$-counting, and myocardial pieces were histopathologically characterized.

In vivo SPECT/CT imaging in MI rabbits $(\mathrm{n}=10)$ revealed intense uptake of ${ }^{111}$ In-GSAO in the apical area (Fig. 3a). Ex vivo SPECT/CT and planar imaging (Fig 3b) of explanted hearts confirmed intense apical radiotracer uptake. $\gamma$-counting confirmed that the ${ }^{111} \mathrm{In}$-GSAO uptake in the myocardial infarct was markedly higher than the ${ }^{111}$ InGSAO uptake in the remote region $(1.10 \pm 0.45 \% \mathrm{ID} / \mathrm{g}$ vs $0.03 \pm$ $0.01 \% \mathrm{ID} / \mathrm{g}, \mathrm{P}=0.005$, Fig. $3 \mathrm{c}$ ).

To study the specificity of ${ }^{111}$ In-GSAO, the myocardial uptake of negative control compound GSCA was evaluated in acute MI rabbits $(\mathrm{n}=5)$. In vivo (Fig. 3a) and ex vivo SPECT/CT imaging and planar imaging (Fig. 3b) revealed very low cardiac uptake of GSCA; only slightly increased infarct uptake was observed. This was confirmed by $\gamma$-counting; when compared with ${ }^{111} \mathrm{In}$-GSAO, ${ }^{111} \mathrm{In}$-GSCA uptake in the infarct area was significantly lower $(0.07$ $\pm 0.03 \% \mathrm{ID} / \mathrm{g}, \mathrm{P}=0.002$, Fig 3c). Although low, GSCA uptake in the infarct area was higher than in the remote area $(0.03 \pm 0.01 \% \mathrm{ID} / \mathrm{g}, \mathrm{P}$ $=0.043)$. This demonstrated that the trivalent arsenical group was necessary for targeting of dying cells. Moreover, myocardial ${ }^{111}$ InGSAO uptake in an unmanipulated control animal was similar to ${ }^{111}$ In-GSAO uptake in the spared myocardium of the MI rabbits $(0.01 \% \mathrm{ID} / \mathrm{g}$ vs $0.03 \pm 0.01 \% \mathrm{ID} / \mathrm{g})$, further supporting specificity of GSAO.

To evaluate localization of cardiac ${ }^{111}$ In-GSAO uptake, three of ten animals receiving ${ }^{111} \mathrm{In}-\mathrm{GSAO}$ also received the myocardial perfusion tracer ${ }^{99 \mathrm{~m}} \mathrm{Tc}$-sestamibi (MIBI), immediately after coronary ligation. Serial in vivo SPECT/CT imaging confirmed that ${ }^{111}$ In-GSAO uptake was localized in the infarct zone as identified by ${ }^{99 \mathrm{~m}} \mathrm{Tc}-\mathrm{MIBI}$ perfusion defect. Similarly, ex vivo serial SPECT/CT imaging and serial planar imaging (Fig. 3d) revealed uptake of ${ }^{111}$ In-GSAO in the infarct zone as shown by ${ }^{99 \mathrm{~m}} \mathrm{Tc}-\mathrm{MIBI}$ perfusion defect. In each animal, $\gamma$ Counting of ${ }^{99 \mathrm{~m}} \mathrm{Tc}-\mathrm{MIBI}$ and ${ }^{111} \mathrm{In}-\mathrm{GSAO}$ in myocardial sections confirmed increased uptake of ${ }^{111}$ In-GSAO in the perfusion defects as sections with low MIBI uptake showed increased ${ }^{111} \mathrm{In}-\mathrm{GSAO}$ uptake and vice versa (fig $3 \mathrm{e}$ ). $\gamma$-counting of all myocardial sections from the animals receiving both ${ }^{111} \mathrm{In}-\mathrm{GSAO}$ and ${ }^{99 \mathrm{~m}} \mathrm{Tc}-\mathrm{MIBI}$ revealed an inverse correlation between ${ }^{111}$ In-GSAO uptake and ${ }^{99 \mathrm{~m}}$ Tc-MIBI uptake $(\rho=-0.70, \mathrm{P}<0.01$, Fig $3 \mathrm{f})$.

Of ten animals receiving ${ }^{111} \mathrm{In}-\mathrm{GSAO}$, three also received ${ }^{99 \mathrm{~m}} \mathrm{Tc}$ AA5. Serial in vivo SPECT/CT imaging with ${ }^{111} \mathrm{In}-\mathrm{GSAO}$ and ${ }^{99 \mathrm{~m}} \mathrm{Tc}$ AA5 revealed similar area of increased uptake; ${ }^{111}$ In-GSAO uptake was more intense than ${ }^{99 \mathrm{~m}}$ Tc-AA5 uptake. This was confirmed by $e x$ vivo serial SPECT/CT imaging and ex vivo serial planar imaging (Fig. 3g). Quantitative tracer uptake paralleled the imaging data. In each animal, in sections with increased ${ }^{99 m}$ Tc-AA5 uptake, ${ }^{111}$ InGSAO uptake was also increased (fig. $3 \mathrm{~h}$ ). Using all myocardial sections in all animals receiving ${ }^{111} \mathrm{In}-\mathrm{GSAO}$ and ${ }^{99 \mathrm{~m}} \mathrm{Tc}-\mathrm{AA} 5$, a strong correlation between uptake of the radiotracers was observed $(\rho=0.82$, $\mathrm{P}<0.01$, Fig 3i). Like the fluorescence experiments, this supports that secondary necrosis after apoptotic signaling may be the dominant mode of cell death in myocardial infarction. The ${ }^{111}$ In-GSAO uptake in the infarct zone was higher than ${ }^{99 \mathrm{~m}}$ Tc-AA5 uptake, although the results did not reach statistical significance, due to the small sample size $(1.10 \pm 0.45 \% \mathrm{ID} / \mathrm{g}$ vs $0.47 \pm 0.23 \%, \mathrm{P}=0.47)$. Uptake in the remote area was low for both tracers $(0.03 \pm 0.01 \% \mathrm{ID} / \mathrm{g}$ vs $0.04 \pm$ $0.02 \% \mathrm{ID} / \mathrm{g}, \mathrm{P}=\mathrm{NS}$ ).

After $\gamma$-counting myocardial pieces from infarct, border and remote zones were histologically characterized. Representative examples of the stainings are given in figure 4a. H\&E staining revealed hypereosinophilic change and contraction band necrosis in the myocardial sections from the infarct area; the morphological changes were less frequent in the border zone. The remote area was morphologically normal. The rate of apoptosis as shown by terminal deoxyribonucleotide transferase TdT-mediated nick-end labeling (TUNEL) staining (\%area positive, \%AP) was higher in the infarct $(2.83 \pm 1.42 \% \mathrm{AP})$ and border zone $(2.55 \pm 1.84 \% \mathrm{AP})$ than in the remote zone $(0.02 \pm 0.0 \% \mathrm{AP}, \mathrm{P}<0.001$ for both). The \%caspase positive area was higher in the infarct $(1.04 \pm 0.48 \% \mathrm{AP})$ than in the remote zone $(0.46 \pm 0.48 \% \mathrm{AP}, \mathrm{P}=0.054) .{ }^{111} \mathrm{In}-\mathrm{GSAO}$ uptake in the myocardial sections showed a direct correlation with TUNEL $(\rho$ : 0.743, $\mathrm{P}<0.001$, Fig. 4 b) and Caspase-3 $(\rho=0.533, \mathrm{P}<0.001$, Fig. 4c) stains, further supporting that necrosis as shown by GSAO is secondary after apoptotic signaling. 

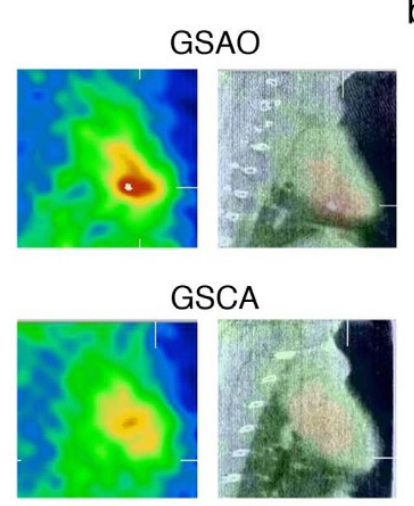

GSCA

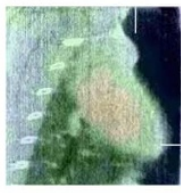

d

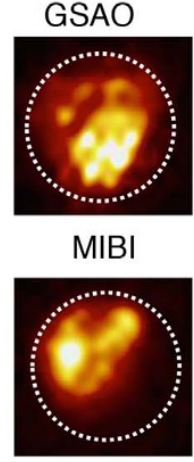

e

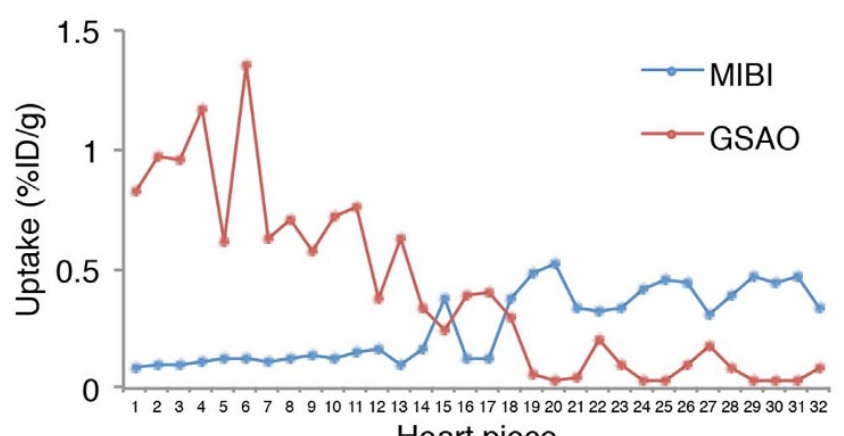

Heart piece

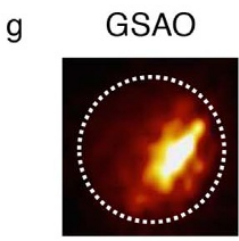

AA5

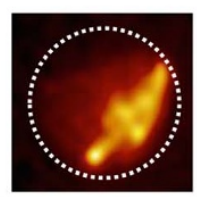

b
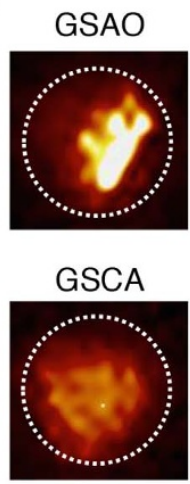

f

i

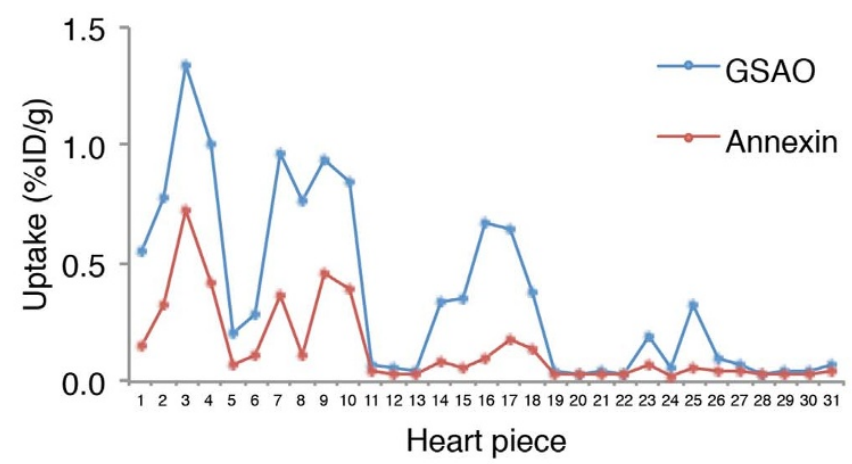

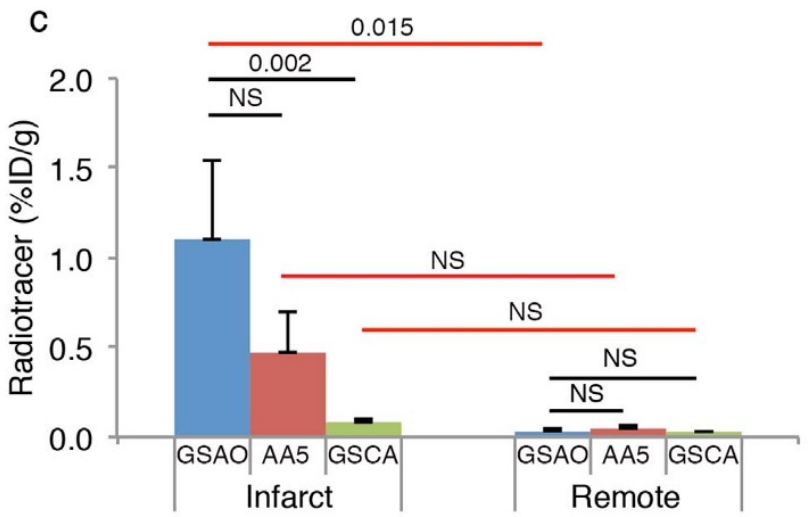
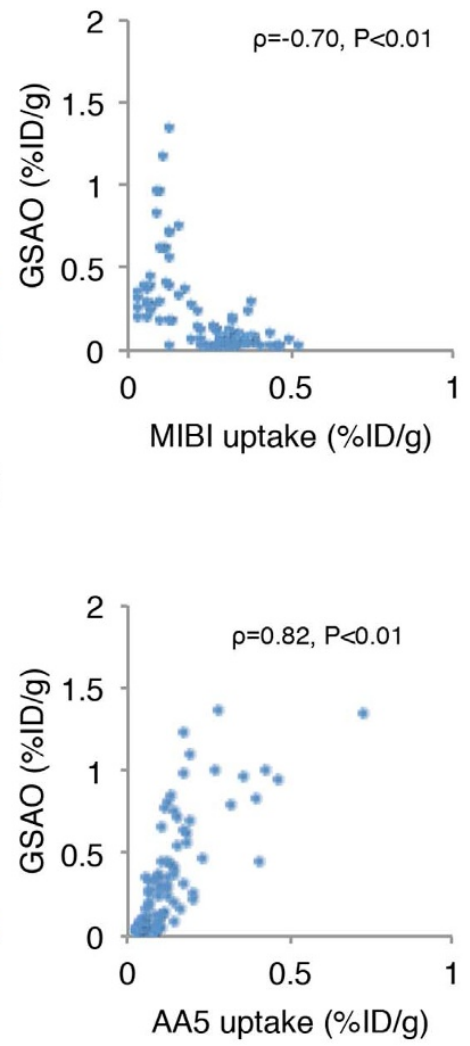

Figure $3 \mid{ }^{111}$ In-GSAO imaging, quantification and comparison with ${ }^{99 \mathrm{~m}} \mathrm{Tc}$-Annexin A5 and ${ }^{99 \mathrm{~m}} \mathrm{Tc}-\mathrm{MIBI}$ in rabbits with acute myocardial infarction. (a) In vivo SPECT (left panels), and fused SPECT/CT images (right panels) in acute MI rabbits revealed intense ${ }^{111}$ In-GSAO uptake (top) and only modest uptake of radiotracer control compound ${ }^{111}$ In-GSCA (bottom). (b) Ex vivo planar images confirmed intense ${ }^{111}$ In-GSAO uptake (top) and modest ${ }^{111}$ InGSCA (bottom) uptake. (c) $\gamma$-counting of myocardial sections confirmed ${ }^{111}$ In-GSAO uptake in infarct was higher than in remote area and higher than ${ }^{111} \mathrm{In}$-GSCA uptake in infarct. Although low, ${ }^{111} \mathrm{In}$-GSCA uptake in infarct was higher than remote. Difference Between ${ }^{111} \mathrm{In}-\mathrm{GSAO}$ and ${ }^{99 \mathrm{~m}} \mathrm{Tc}-\mathrm{AA} 5$ uptake did not reach significant uptake. Black horizontal lines denote Kruskal-Wallis ANOVAs + Bonferroni correction, and red lines denote Wilcoxon signed rank tests + Bonferroni correction. (d) Serial imaging revealed that ${ }^{111}$ In-GSAO uptake (top) was predominantly localized in the region of the ${ }^{99 m}$ Tc-MIBI perfusion defect (bottom). (e) $\gamma$-counting of myocardial sections showed high ${ }^{111}$ In-GSAO uptake in sections with low MIBI uptake and vice versa. (f) A significant inverse Spearman's correlation between uptake of ${ }^{111} \mathrm{In}-\mathrm{GSAO}$ and ${ }^{99 \mathrm{~m}} \mathrm{Tc}-\mathrm{MIBI}$ was observed. (g) Serial planar imaging in acute MI rabbit demonstrates similar uptake region and higher uptake of ${ }^{111}$ In-GSAO (top) when compared with ${ }^{99 m}$ Tc-AA5 (bottom). (h) $\gamma$-counting of myocardial sections showed high ${ }^{111} \mathrm{In}$-GSAO uptake in sections with high ${ }^{99 \mathrm{~m}} \mathrm{Tc}$-AA5 uptake and vice versa (i). A significant Spearman's correlation between uptake of ${ }^{111} \mathrm{In}-\mathrm{GSAO}$ and ${ }^{99 \mathrm{~m}} \mathrm{Tc}-\mathrm{AA} 5$ was observed.

The non-target organ distribution of ${ }^{111}$ In-GSAO and ${ }^{111}$ In-GSCA demonstrated kidney to be the major organ of radiation burden and urine major route of excretion; all other organs revealed minimum burden for both radiotracers (Supplementary Fig. 1a). Serial blood samples from six animals revealed bi-exponential blood clearance with an initial fast component $\mathrm{T}_{1 / 2} \alpha$ of $5.3 \mathrm{~min}$ followed by a slower component $\mathrm{T}_{1 / 2} \beta$ of $9.7 \mathrm{~h}$; the plateau phase was approached at 15 min (Supplementary Fig. 1b).

In vivo SPECT/CT imaging in mice after acute $\mathrm{MI}(\mathrm{n}=6)$ demonstrated high ${ }^{111}$ In-GSAO uptake; the use of CT allowed precise localization of the radioactivity in the infarcted region of the heart (Fig. 5a,b). Specific apical uptake was confirmed by ex vivo 
a

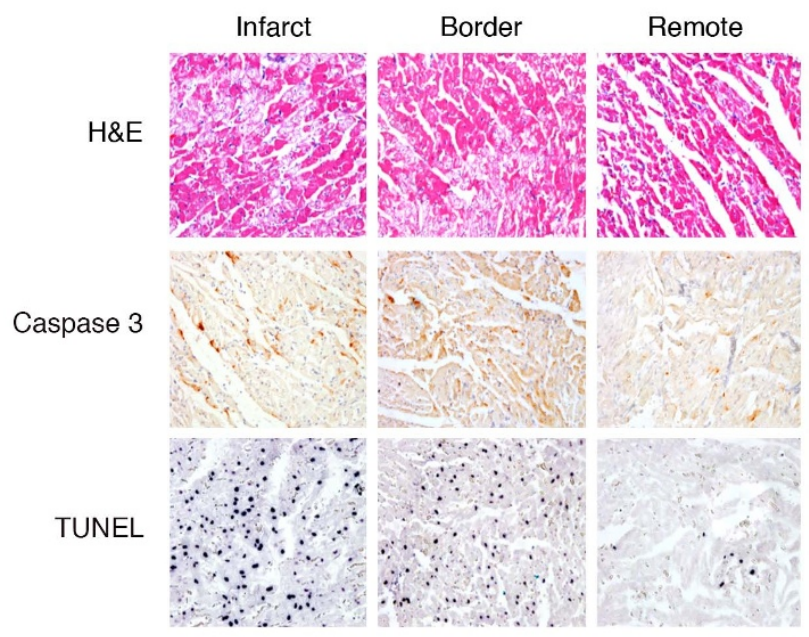

b

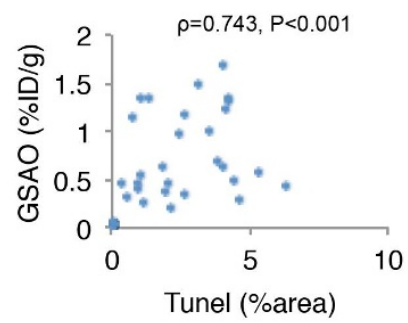

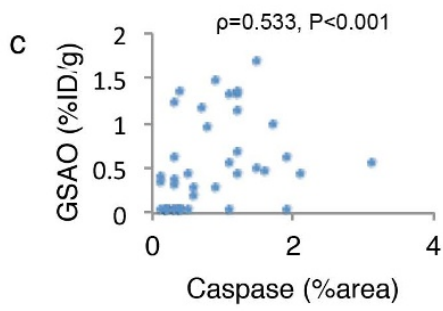

Figure $4 \mid$ Histopathological characterization of ${ }^{111}$ In-GSAO uptake in rabbit heart after experimental acute myocardial infarction. H\&E staining demonstrates clear signs of tissue damage in infarct and border zone, whereas the remote zone is morphologically normal. TUNEL and Caspase-3 stains demonstrate substantial apoptosis in the infarct zone, whereas positive cells are rarely seen in the remote area. Significant Spearman's Rank correlations between ${ }^{111}$ In-GSAO uptake and apoptotic signaling as shown by TUNEL (b) and Caspase-3 (c) stains were observed.

SPECT/CT and planar imaging (Fig. 5c). Imaging experiments revealed absence of specific ${ }^{111}$ In-GSAO uptake in control animals. ${ }^{111} \mathrm{In}-\mathrm{GSAO}$ uptake in the infarct area $(2.56 \pm 2.75 \% \mathrm{ID} / \mathrm{g})$ was markedly higher than in remote myocardium $(0.48 \pm 0.22 \% \mathrm{ID} / \mathrm{g}, \mathrm{P}=$ 0.028 ) and 20-fold higher than apical uptake in unmanipulated control animals $(n=5,0.13 \pm 0.02 \% \mathrm{ID} / g ; \mathrm{p}=0.004$, Fig. $5 \mathrm{~d})$.

Radionuclide imaging in a post-MI HF model. ${ }^{111}$ In-GSAO uptake was also evaluated in post-MI HF mouse model, in which the LCA territory was not reperfused. Subgroups underwent imaging experiments at different time points. Also, a disease control group consisting of animals that did not undergo infarction surgery before ${ }^{111}$ In-GSAO imaging, and a radiotracer control group consisting of animals receiving negative control compound ${ }^{111}$ In-GSCA imaging at 2 weeks post-MI were used.

${ }^{111}$ In-GSAO uptake in the HF mice was markedly lower than in AMI mice, and could not be detected by in vivo SPECT/CT imaging. Quantification of radionuclide uptake in short axis slices confirmed that infarct uptake of ${ }^{111} \mathrm{In}-\mathrm{GSAO}$ in mice at 2 weeks postMI $(n=6)$ was higher than in the five disease control animals that did not undergo infarction surgery $(0.42 \pm 0.17 \% \mathrm{ID} / \mathrm{g}$ vs $0.13 \pm$ $0.02 \% \mathrm{ID} / \mathrm{g}, \mathrm{P}=0.025$, Fig 6 ); remote uptake was not statistically different $(0.25 \pm 0.12 \% \mathrm{ID} / \mathrm{g}$ vs $0.14 \pm 0.04 \% \mathrm{ID} / \mathrm{g}, \mathrm{P}=1.0$, Fig 6$)$. Moreover, ${ }^{111}$ In-GSAO uptake at $2 \mathrm{w}$ post-MI was significantly higher than uptake of the negative control compound ${ }^{111}$ In-GSCA in the infarct and remote area $(0.04 \pm 0.02 \% \mathrm{ID} / \mathrm{g}, \mathrm{P}<0.001$ and $0.04 \pm$ $0.03 \% \mathrm{ID} / \mathrm{g}, \mathrm{P}=0.004$, respectively).

Moreover, when compared with uptake at 2 weeks post-MI, ${ }^{111} \mathrm{In}$ GSAO uptake showed a trend of decline at 4 weeks $(n=6$, infarct $0.28 \pm 0.09 \% \mathrm{ID} / \mathrm{g}, \mathrm{P}=1.0$; remote $0.22 \pm 0.04 \% \mathrm{ID} / \mathrm{g}, \mathrm{P}=1.0$, Fig 6) and 12 weeks $(\mathrm{n}=8$, infarct: $0.19 \pm 0.07 \% \mathrm{ID} / \mathrm{g}, \mathrm{P}=0.332$; remote: $0.15 \% \pm 0.1 \% \mathrm{ID} / \mathrm{g}, \mathrm{P}=1.0$, Fig 6 ) although differences were not statistically significant.

\section{Discussion}

Here we demonstrate cell death imaging in acute myocardial infarction using ${ }^{111}$ In-GSAO. The specificity of the radiotracer was confirmed by the localization of ${ }^{111}$ In-GSAO in the infarct zone as shown by ${ }^{99 \mathrm{~m}} \mathrm{Tc}$-MIBI. This was further supported by the lack of uptake of fluorescently labeled GSAO in sham-operated animals outside of the regions directly damaged by the suture. The lack of GSCA uptake in our radionuclide studies confirmed that the trivalent arsenic group on GSAO is responsible for its targeting characteristics ${ }^{15,29,30}$. The radiotracer showed favorable pharmacokinetic profile with rapid blood clearance and low background uptake in most organs.

In the 1990's evidence of apoptotic signaling in myocardial infarction accumulated ${ }^{19-22}$, sparking the discussion over the relative importance of the apoptotic and necrotic forms of cell death. In $2000,{ }^{99 m}$ Tc-AA5 SPECT in patients with acute myocardial infarction revealed intense uptake in the entire region of the perfusion defect ${ }^{5}$. This observation was provocative as ${ }^{99 \mathrm{~m}} \mathrm{Tc}-\mathrm{AA} 5$ was believed to identify apoptotic cell death but the infarct area was traditionally
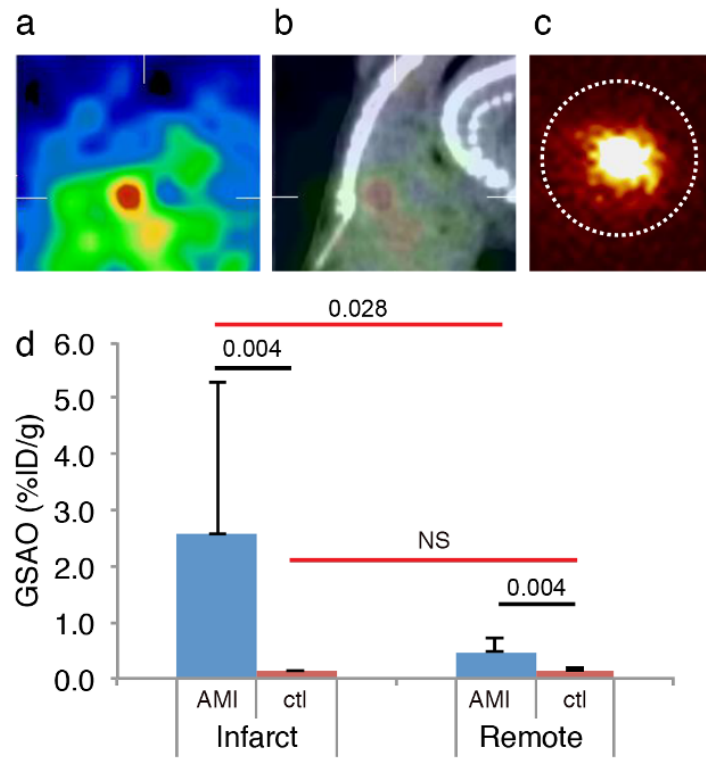

Figure 5 | In vivo and ex vivo ${ }^{111}$ In-GSAO imaging and quantification in mice with acute myocardial infarction. In vivo SPECT (a), and fused SPECT/CT images (b) in acute MI rabbits revealed intense cardiac ${ }^{111} \mathrm{In}$ GSAO uptake. (c) Ex vivo planar images confirmed intense ${ }^{111} \mathrm{In}-\mathrm{GSAO}$ uptake. (d) $\gamma$-counting of myocardial sections confirmed ${ }^{111}$ In-GSAO uptake in infarct (apex) was higher than manyfold higher than in remote area and higher than apical ${ }^{111}$ In-GSAO uptake in unmanipulated control animals. Black horizontal lines denote Mann-Whitney U tests and red lines denote Wilcoxon signed rank tests. 


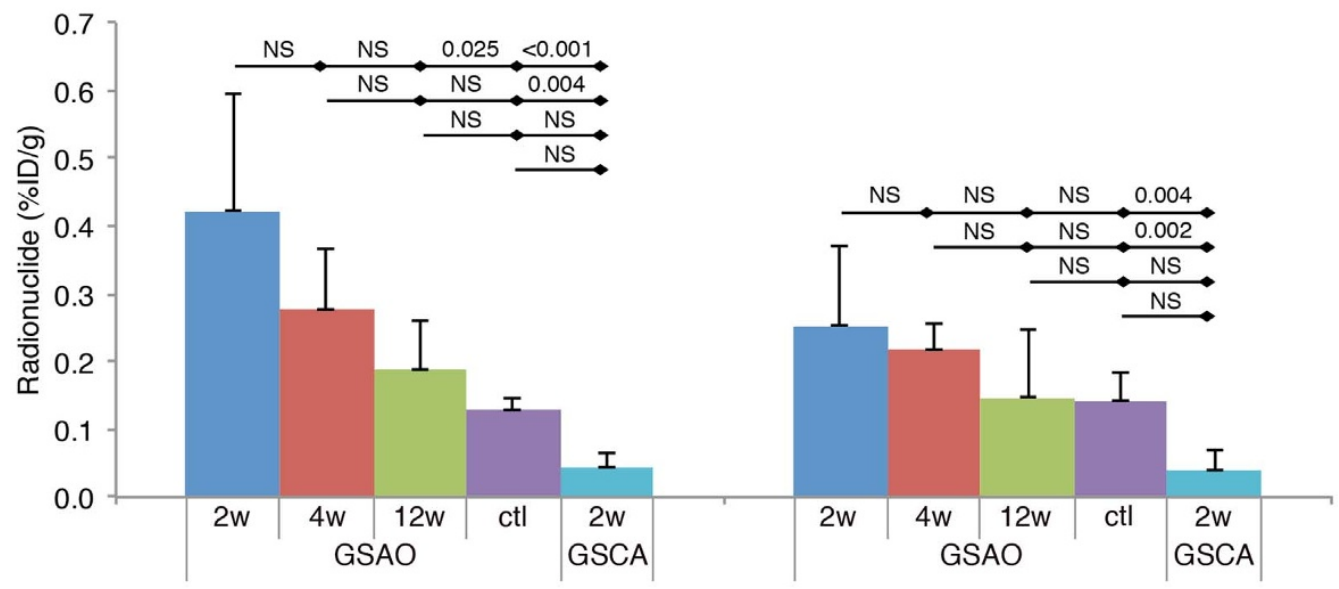

Figure 6 Quantification of ${ }^{111}$ In-GSAO uptake in mice with chronic myocardial infarction. $\Gamma$-counting revealed ${ }^{111}$ In-GSAO uptake in the infarct area at 2 weeks; uptake was significantly higher than ${ }^{111} \mathrm{In}-\mathrm{GSAO}$ uptake in unmanipulated control animals and ${ }^{111}$ In-GSCA uptake at $2 \mathrm{w}$ post-MI in controls. A trend of declining ${ }^{111}$ In-GSAO uptake over time was observed, but did not reach statistical significance. ${ }^{111}$ In-GSAO uptake in remote area was higher than ${ }^{111}$ In-GSCA uptake at $2 \mathrm{w}$ post-MI. Results of Kruskal-Wallis ANOVAs + Bonferroni correction are shown.

expected to be necrotic. It was subsequently proposed that the ischemic insult may be initiated as apoptosis but conclude with secondary necrosis. Ischemic loss of ATP production during myocardial infarction would preclude completion of the energy-dependent apoptosis program ${ }^{23}$. Restoration of blood flow by reperfusion may either interrupt the apoptotic process to allow cell salvage $e^{6,24}$ or resume the process of apoptosis in critically damaged cells ${ }^{20,22}$. The latter may even be augmented further by production of radical oxygen species ${ }^{25}$, or intracellular calcium overload ${ }^{26}$. These changes may also contribute to secondary necrosis by opening of the mitochondrial permeability transition pore ${ }^{27-30}$. It is conceivable that the apoptotic process initiated by noxious stimuli may not follow classical picture of physiologic apoptosis observed during normal turnover of skin or mucosal cells.

The feasibility of cell death imaging using fluorescently labeled and radiolabelled GSAO has recently been demonstrated in tumorbearing mice and mice with experimental brain trauma ${ }^{13-15}$. GSAO accumulation was characterized by fluorescent microscopy in various cell models of apoptotic and in explanted brains and tumors after in vivo GSAO administration. GSAO was observed intracellularly and colocalized with propidium iodide and Sytox blue, standard markers of membrane disruption, which supported GSAO uptake in secondarily necrotic cells. Similarly, our fluorescent experiments showed that GSAO accumulated intracellularly and only occurred in AA5 + cells, thereby supported the notion that necrosis followed apoptotic signaling in the setting of acute myocardial infarction.

In vivo and ex vivo ${ }^{111}$ In-GSAO imaging in rabbits and mice with acute myocardial infarction showed intense dual serial imaging with ${ }^{99 \mathrm{~m}} \mathrm{Tc}-\mathrm{AA} 5$ and ${ }^{111} \mathrm{In}-\mathrm{GSAO}$ in rabbits after acute myocardial ischemia and reperfusion revealed the same area of uptake. At a segment level, ${ }^{111}$ In-GSAO uptake showed the same pattern as ${ }^{99 \mathrm{~m}} \mathrm{Tc}-\mathrm{AA} 5$ and a strong correlation between uptakes of the two tracers was shown. In addition, ${ }^{111}$ In-GSAO correlated with presence of Caspase- 3 and TUNEL staining, classic markers of apoptosis. This shows that not only does secondary necrosis after apoptotic signaling occur in myocardial ischemia and reperfusion, it may play a dominant role. As in rabbits, high ${ }^{111} \mathrm{In}-\mathrm{GSAO}$ uptake was seen in mice with acute myocardial infarction. However, ${ }^{111} \mathrm{In}-\mathrm{GSAO}$ uptake in post-myocardial infarction HF model in mice was too low to be detected by microSPECT/CT imaging. The most important explanation for this is the lower rate of necrotic cell death in heart failure ${ }^{31-33}$. It is tempting to speculate that the more benign circumstances such as lesser energy depletion and ROS cause lower transition from apoptosis to necrosis in this setting. In fact, the apoptotic process may remain suspended in chronic heart failure ${ }^{34}$.

A number of imaging tracers for myocardial necrosis have been clinically evaluated. Like ${ }^{111}$ In-GSAO, most tracers exploited membrane disruption, the hallmark of necrosis as a target. Tc-99 labelled antimyosin antibody was the most widely studied necrosis tracer. Antimyosin antibody imaging has been successfully employed for the detection of myocardial necrosis associated with myocardial infarction ${ }^{9}$, myocarditis ${ }^{9}$, heart failure ${ }^{12}$ and cardiac allograft rejection $^{12}$. However, because of long circulation time of the radiolabelled antibody, imaging was not feasible for up to 6-12 hours after administration of the agent. ${ }^{99 \mathrm{~m}} \mathrm{Tc}$-pyrophosphate on the other hand, showed maximal myocardial uptake at 24-72 hours after injection, although necrosis imaging at 3 hours post-administration was feasible, maximum myocardial uptake was at 24-72 h. Also, this tracer required residual blood flow, which precluded uptake in the infarct center ${ }^{35} .{ }^{99 m}$ Tc-glucarate can be used to necrotic cells in myocardial infarction ${ }^{11,36}$, and has good imaging characteristics but in the setting of myocardial infarction, use is limited to a clinical window of $>9$ hours after onset as its target, histone bodies, quickly wash out of the tissue ${ }^{11}$. Late gadolinium-enhanced MRI has also been used to assess cardiac cell death; the contrast medium accumulates in necrotic cells through the disrupted membranes. However, its specificity is limited, as in all instances of increased extracellular space such as cardiac edema, fibrosis, sarcoidosis and amyloidosis ${ }^{37-39} \cdot{ }^{111} \mathrm{In}-\mathrm{GSAO}$ appears to have advantages over the previously evaluated cell death imaging techniques. First, ${ }^{111}$ In-GSAO requires membrane disruption, the hallmark of necrotic or late-apoptotic cell death, for reaching its intracellular targets. Therefore it does not suffer from the lack of specificity of late-gadolinium enhanced MRI. Moreover, its rapid blood clearance results in feasibility of early imaging of cell death using ${ }^{111} \mathrm{In}-\mathrm{GSAO}$. This gives ${ }^{111} \mathrm{In}-\mathrm{GSAO}$ an advantage over ${ }^{99 \mathrm{~m}} \mathrm{Tc}$ antimyosin and ${ }^{99 m}$ Tc-pyrophosphate. HSP90, the main target of GSAO, functions in complexes. This may allow for targeting over a longer time period, giving ${ }^{111} \mathrm{In}-\mathrm{GSAO}$ an edge over ${ }^{99 \mathrm{~m}} \mathrm{Tc}$-glucarate. However, this has to be evaluated in follow-up studies.

Micro-SPECT/CT imaging using ${ }^{111}$ In-GSAO can be used to visualize necrotic cell death in acute myocardial infarction. The uptake in the HF model was too low to allow imaging. Because the GSAO uptake reflects membrane permeabilization, and occurs predominantly in AA5 positive cells, we propose that secondary necrosis is a dominant mode of cell death in the setting of myocardial ischemia and reperfusion. 
distribution. To correct for the radioactive decay and permit calculation of the concentration of radioactivity as a fraction of the administered dose, aliquots of the injected dose were counted simultaneously. For evaluation of infarct ${ }^{111}$ In-GSAO uptake in rabbits, the section with the highest ${ }^{111} \mathrm{In}-\mathrm{GSAO}$ uptake in the infarct area was used of each animal, and for evaluation of remote ${ }^{111}$ In-GSAO uptake, the section with the lowest ${ }^{111}$ In-GSAO uptake in the remote area was selected. In all mice, the apical slice was used for evaluation of infarct uptake, the middle slice for uptake in the border zone and the basal slice for remote uptake.

Ethical statement. The experimental protocols followed the Guidelines for the Care and Use of Laboratory Animals established by the National Institutes of Health (NIH Publication No. 85-23, revised 1996) and was approved by the Institutional

Laboratory Animal Care and Use Committees at the University of California, Irvine and University of Maastricht, Maastricht, The Netherlands.

Experimental myocardial infarction in mice. For fluorescence experiments, C57Bl6/j (Age: 3 months, weight: $\sim 50$ g, Jackson Laboratories, Sacramento, CA) and for radionuclide studies Swiss-Webster mice (Age: 3 months, weight $\sim 50$ g, Charles River, Wilmington, MA) were used. MI was induced under isoflurane anesthesia (23\%) using a stereomicroscope (Leica MZ FL III, Leica, Switzerland) as described previously ${ }^{40}$. Animals were placed on a heating pad in the supine position, intubated under direct laryngoscopy, and mechanically ventilated using a small animal respirator (tidal volume, $1.0 \mathrm{ml}$; rate, 120 breaths $/ \mathrm{min}$, Harvard Apparatus, Holliston, MA). After a minimum thoracotomy, the anterior descending branch of the left coronary artery was ligated with a 6.0 -silk suture 3 to $4 \mathrm{~mm}$ below the tip of the left atrium. Successful ligation was verified by visual inspection of the LV apex for myocardial blanching, indicating interruption in coronary flow. For induction of acute MI experiments, the suture was removed after 30 minutes to induce reperfusion. The sham operation was identical, but the coronary artery was not ligated. Acute MI and sham animals remained under anesthesia for ensuing radionuclide imaging or fluorescence experiments. For induction of chronic MI, the ligation was not released. In these animals, the chest cavity was closed in layers with 6.0 -silk and the skin was closed with 4.0-silk sutures. Thereafter, animals were gradually weaned from the respirator and put back into their cages until imaging procedure.

Experimental acute myocardial infarction in rabbits. Acute experimental MI was induced by the occlusion of left anterior descending coronary artery (LAD) in New Zealand White male rabbits (weight, $3.0-3.5 \mathrm{~kg}$ ) as described previously ${ }^{24,41}$. All rabbits were anesthetized with a mixture of ketamine and xylazine $(100 \mathrm{mg} / \mathrm{ml}, 10: 1$ $\mathrm{vol} / \mathrm{vol} ; 2.0$ to $3.0 \mathrm{ml}$ subcutaneously). Surgical tracheostomy was performed, and ventilation was maintained with a volume-cycled rodent respirator (Harvard Apparatus, Holliston, MA) provided positive pressure ventilation at $50 \mathrm{~mL} /$ cycle and a respiratory rate of $50 \mathrm{cycles} / \mathrm{min}$. After surgical procedure, anesthesia was maintained on 3-4\% isoflurane Briefly, the heart was exposed through parasternal thoracotomy, and the pericardium was fenestrated. The LAD was identified and a monofilament suture was placed at the site. The LAD was occluded by tightening the snare created by passing suture through a polyethylene tube. The snare was removed after 40 minutes of occlusion to induce reperfusion. Lead II or III of the electrocardiogram was continuously monitored during the experiments to confirm myocardial ischemia. Animals remained anesthetized for ensuing radionuclide imaging experiments.

Fluorescence experiments in mice. Acute MI mice and sham-operated mice were injected with Cy5.5-labelled GSAO at 5 minutes before ischemia and 1 hour before sacrifice, respectively. All mice received Oregon Green-labeled AA5 10 minutes before sacrifice. Of each fluorescent probe a dosage of $2.5 \mathrm{mg} / \mathrm{kg}$ was used. Acute MI animals were sacrificed after $30 \mathrm{~m}$ reperfusion, sham mice at 1 hour after sacrifice hearts were snap-frozen in liquid nitrogen, $7 \mu \mathrm{m}$ thick frozen sections were obtained, dried and mounted in 4,6-diamino-2-phenylindole (DAPI) containing medium and examined with a confocal scanning laser microscope (Bio-Rad) equipped with a krypton/argon mixed gas laser (Ion Laser Technology).

In vivo and ex vivo imaging protocols mice and rabbits. For mice and rabbits, the same imaging procedure was followed. In vivo SPECT imaging was performed at 3 hours after radiotracer administration using a dual-head micro-SPECT $\gamma$-camera combined with micro-CT (X-SPECT, Gamma Medica, Inc., Northridge, CA) under isoflurane anesthesia. SPECT images of the heart were acquired in a $64 \times 64$ matrix at 32 steps at 60 seconds per step with a $247 \mathrm{keV}$ photo peak of ${ }^{111} \mathrm{In}$ with $15 \%$ windows using a medium energy parallel-hole collimator. In the animals also undergoing

${ }^{99 \mathrm{~m}} \mathrm{Tc}-\mathrm{MIBI}{ }^{99 \mathrm{~m}} \mathrm{Tc}-\mathrm{AA} 5$, this was followed by imaging using the same protocol, at a $140 \mathrm{keV}$ photo peak of ${ }^{99 \mathrm{~m}} \mathrm{Tc}$ with $15 \%$ windows using a low-energy, high-resolution parallel-hole collimator. Gating was not performed. After SPECT imaging acquisition, a CT scan was acquired using an X-ray tube operating at $50 \mathrm{kVp}$ and $0.6 \mathrm{~mA}$. Images were acquired for 2.5 seconds per view for 256 views in $360^{\circ}$ rotation. After transferring to $256 \times 256$ matrix, the SPECT images and CT studies were fused. After imaging, anesthetized animals were terminated by heart explantation and $e x$ vivo SPECT/CT excised heart was performed using the same protocols but at 45 seconds per step for SPECT. Thereafter, planar ${ }^{111}$ In-GSAO imaging of the hearts was performed for 15 minutes, followed by ${ }^{99 \mathrm{~m}} \mathrm{Tc}$-MIBI or ${ }^{99 \mathrm{~m}} \mathrm{Tc}-\mathrm{AA} 5$ planar imaging in GSAO-MIBI and GSAO-AA5 animals. After ex vivo imaging, rabbit hearts were cut into 4 short axis slices and further divided into 31-32 pieces. Mice hearts were cut in 3 short-axis slices. All sections were weighed, and $\gamma$-counted in an automatic well-type $\gamma$-counter (Perkin Elmer Wallac Inc., Gaithersburg, MD) for calculation of the percent total injected dose per tissue weight (\%ID/g) uptake. Tissue samples of the main organs were used for calculation of the $\% \mathrm{ID} / \mathrm{g}$ uptake to evaluate the bio
Pharmacokinetic studies. The pharmacokinetic parameters were derived using the KINFIT module of the MW/PHARM computer program package (Version 3.60, MediWare, Groningen, The Netherlands $)^{42}$. The data, consisting of the blood concentration of ${ }^{111}$ In radioactivity or GSAO versus time, were analyzed by nonlinear regression analysis using a least-squares weighted simplex algorithm, with data weighted with the reciprocal of the observed value.

Histological, immunohistochemical evaluation. After $\gamma$-counting of ${ }^{111}$ In-GSAO uptake, myocardial pieces of seven rabbits were processed for histopathologic characterization. Of each animal, two pieces from each region (infarct, border zone, remote) were selected.

The myocardial pieces were fixed overnight 4\% paraformaldehyde in PBS (pH 7.4 at $4{ }^{\circ} \mathrm{C}$ ), and stored in PBS with $0.02 \%$ sodium azide at $4{ }^{\circ} \mathrm{C}$ until used. The specimens were further processed by dehydration in a graded series of ethanol for paraffinembedding. The blocks were cut in 5 - $\mu \mathrm{m}$ thick sections, floated on a water bath containing deionized water $\left(43^{\circ} \mathrm{C}\right)$, and transferred to vectabond (Vector

Laboratories Burlingame, California) reagent-treated slides (Vector SP-1800, Vector Laboratories), dried overnight, and stored until ready for use. Sections were deparaffinised by heating $\left(25\right.$ minutes at $\left.56^{\circ} \mathrm{C}\right)$ and dehydration using xylene and graded series of ethanol. Tissue sections were stained with standard haematoxylin \& eosin and Masson's trichrome staining. For immunohistochemical characterization, adjacent sections were incubated with primary antibodies against Caspase-3. After washing with PBS, sections were incubated with a biotinylated secondary antibody. The presence of apoptotic cells was further evaluated using TUNEL staining as described previously. Briefly: exposed DNA fragments were labeled with biotinylated nucleotides (dNTPs) and TdT for $1 \mathrm{~h}$ at $37^{\circ} \mathrm{C}$ after blocking of endogenous perosidase activity using $0.3 \%$ hydrogen peroxide and incubation with proteinase $\mathrm{K}^{41}$. For color reactions, sections were incubated with diaminobenzidine. For the assessment of the immunopositive area, stained tissue sections were observed under appropriate magnification (Carl Zeiss, Thornwood, New York), and the images were captured with a high-resolution digital camera (Axiocam, 1,300 $\times 1,030$ pixels, Carl Zeiss) using Axiovision 3.1 software. Digital images were analyzed using Image-Pro Plus 5.0 (Media Cybernetics, Bethesda, Maryland).

Statistical analysis. All results are presented as the mean \pm SD. In most cases, the data did not meet the assumptions of parametric tests. For consistency, nonparametric tests were used for all comparisons. Related samples were compared using Wilcoxon Signed Rank test (two groups) or Friedman's Two Way ANOVA followed by post-hoc Wilcoxon Signed-Rank tests and Bonferroni correction for pair-wise significance (more than two groups). Unrelated samples were compared using Mann Whitney U tests (two groups) or Kruskal-Wallis ANOVA followed by MannWhitney $U$ tests and Bonferroni Correction for pair-wise significance (more than two groups). When the Kruskal-Wallis test revealed no significant differences among groups, no post-hoc tests were performed and $\mathrm{P}=\mathrm{NS}$ was reported. Bonferroni correction was also applied to results of multiple Wilcoxon signed rank tests regarding the uptake of radiotracers in infarct vs. remote areas in rabbits (fig 3c). To assess the correlation between ${ }^{111} \mathrm{In}-\mathrm{GSAO}$ uptake and ${ }^{99 \mathrm{~m}} \mathrm{Tc}-\mathrm{AA} 5$ uptake, ${ }^{99 \mathrm{~m}} \mathrm{Tc}$ MIBI uptake and histologic findings (caspase-3 and TUNEL), Spearman's $\rho$ s were

1. Konstantinidis, K., Whelan, R. S. \& Kitsis, R. N. Mechanisms of cell death in heart disease. Arterioscler Thromb Vasc Biol 32, 1552-1562 (2012).

2. Nikoletopoulou, V., Markaki, M., Palikaras, K. \& Tavernarakis, N. Crosstalk between apoptosis, necrosis and autophagy. Biochim Biophys Acta 1833, 3448-3459 (2013).

3. Nicotera, P. \& Melino, G. Regulation of the apoptosis-necrosis switch. Oncogene 23, 2757-2765 (2004)

4. Boersma, H. H. et al. Past, present, and future of annexin A5: from protein discovery to clinical applications. J Nucl Med 46, 2035-2050 (2005).

5. Hofstra, L. et al. Visualisation of cell death in vivo in patients with acute myocardial infarction. Lancet 356, 209-212 (2000).

6. Thimister, P. W. et al. In vivo detection of cell death in the area at risk in acute myocardial infarction. J Nucl Med 44, 391-396 (2003).

7. Narula, J. et al. Annexin-V imaging for noninvasive detection of cardiac allograft rejection. Nat Med 7, 1347-1352 (2001)

8. Kietselaer, B. L. et al. Noninvasive detection of programmed cell loss with $99 \mathrm{mTc}$ labeled annexin A5 in heart failure. J Nucl Med 48, 562-567 (2007).

9. Khaw, B. A. \& Narula, J. Non-invasive detection of myocyte necrosis in myocarditis and dilated cardiomyopathy with radiolabelled antimyosin. Eur Heart J 16 Suppl O, 119-123 (1995).

10. Corbett, J. R. et al. $99 \mathrm{mTc}$-pyrophosphate imaging in patients with acute myocardial infarction: comparison of planar imaging with single-photon calculated. $P$ values of $<0.05$ were considered statistically significant. 
tomography with and without blood pool overlay. Circulation 69, 1120-1128 (1984).

11. Mariani, G. et al. Detection of acute myocardial infarction by $99 \mathrm{mTc}$-labeled Dglucaric acid imaging in patients with acute chest pain. J Nucl Med 40, 1832-1839 (1999).

12. Narula, J. et al. Antimyosin uptake and myofibrillarlysis in dilated cardiomyopathy. J Nucl Cardiol 2, 470-477 (1995).

13. Park, D. et al. Noninvasive imaging of cell death using an Hsp90 ligand. J Am Chem Soc 133, 2832-2835 (2011)

14. Park, D. et al. Optical imaging of treatment-related tumor cell death using a heat shock protein-90 alkylator. Mol Pharm 10, 3882-3891 (2013).

15. Xie, B. W. et al. Optical imaging of cell death in traumatic brain injury using a heat shock protein-90 alkylator. Cell Death Dis 4, e473 (2013).

16. Donoghue, N., Yam, P. T., Jiang, X. M. \& Hogg, P. J. Presence of closely spaced protein thiols on the surface of mammalian cells. Protein Sci 9, 2436-2445 (2000).

17. Dilda, P. J., Ramsay, E. E., Corti, A., Pompella, A. \& Hogg, P. J. Metabolism of the tumor angiogenesis inhibitor 4-(N-(S-Glutathionylacetyl)amino)phenylarsonous acid. J Biol Chem 283, 35428-35434 (2008).

18. Goetz, M. P., Toft, D. O., Ames, M. M. \& Erlichman, C. The Hsp90 chaperone complex as a novel target for cancer therapy. Ann Oncol 14, 1169-1176 (2003)

19. Scarabelli, T. M. et al. Clinical implications of apoptosis in ischemic myocardium. Curr Probl Cardiol 31, 181-264 (2006).

20. Fliss, H. \& Gattinger, D. Apoptosis in ischemic and reperfused rat myocardium. Circ Res 79, 949-956 (1996)

21. Freude, B. et al. Apoptosis is initiated by myocardial ischemia and executed during reperfusion. J Mol Cell Cardiol 32, 197-208 (2000).

22. Gottlieb, R. A., Burleson, K. O., Kloner, R. A., Babior, B. M. \& Engler, R. L. Reperfusion injury induces apoptosis in rabbit cardiomyocytes. J Clin Invest $\mathbf{9 4}$, 1621-1628 (1994)

23. Narula, J. \& Strauss, H. W. Invited commentary: P.S.* I love you: implications of phosphatidyl serine (PS) reversal in acute ischemic syndromes. J Nucl Med $\mathbf{4 4}$ 397-399 (2003).

24. Kenis, H. et al. Annexin A5 uptake in ischemic myocardium: demonstration of reversible phosphatidylserine externalization and feasibility of radionuclide imaging. J Nucl Med 51, 259-267 (2010).

25. Li, C. \& Jackson, R. M. Reactive species mechanisms of cellular hypoxiareoxygenation injury. Am J Physiol Cell Physiol 282, C227-241 (2002).

26. Orrenius, S., Zhivotovsky, B. \& Nicotera, P. Regulation of cell death: the calciumapoptosis link. Nat Rev Mol Cell Biol 4, 552-565 (2003).

27. Halestrap, A. P. Calcium, mitochondria and reperfusion injury: a pore way to die. Biochem Soc Trans 34, 232-237 (2006).

28. Halestrap, A. Biochemistry: a pore way to die. Nature 434, 578-579 (2005).

29. Halestrap, A. P., Gillespie, J. P., O’Toole, A. \& Doran, E. Mitochondria and cell death: a pore way to die? Symp Soc Exp Biol 52, 65-80 (2000).

30. Arbustini, E. \& Narula, J. Cyclosporin a in reperfusion injury: not opening to cell death knocking at the door? Ann Thorac Surg 89, 1349-1351 (2010).

31. Yue, T. L. et al. Possible involvement of stress-activated protein kinase signaling pathway and Fas receptor expression in prevention of ischemia/reperfusioninduced cardiomyocyte apoptosis by carvedilol. Circ Res 82, 166-174 (1998).

32. Condorelli, G. et al. Increased cardiomyocyte apoptosis and changes in proapoptotic and antiapoptotic genes bax and bcl-2 during left ventricular adaptations to chronic pressure overload in the rat. Circulation 99, 3071-3078 (1999).

33. Li, Z., Bing, O. H., Long, X., Robinson, K. G. \& Lakatta, E. G. Increased cardiomyocyte apoptosis during the transition to heart failure in the spontaneously hypertensive rat. Am J Physiol 272, H2313-2319 (1997).

34. Narula, J., Haider, N., Arbustini, E. \& Chandrashekhar, Y. Mechanisms of disease: apoptosis in heart failure--seeing hope in death. Nat Clin Pract Cardiovasc Med 3, 681-688 (2006).

35. De Saint-Hubert, M., Prinsen, K., Mortelmans, L., Verbruggen, A. \& Mottaghy, F. M. Molecular imaging of cell death. Methods (San Diego, Calif.) 48, 178-187 (2009)
36. Narula, J., Petrov, A., Pak, K. Y., Lister, B. C. \& Khaw, B. A. Very early noninvasive detection of acute experimental nonreperfused myocardial infarction with 99mTc-labeled glucarate. Circulation 95, 1577-1584 (1997).

37. Perugini, E. et al. Non-invasive evaluation of the myocardial substrate of cardiac amyloidosis by gadolinium cardiac magnetic resonance. Heart 92, 343-349 (2006).

38. Shimada, T. et al. Diagnosis of cardiac sarcoidosis and evaluation of the effects of steroid therapy by gadolinium-DTPA-enhanced magnetic resonance imaging. Am J Med 110, 520-527 (2001).

39. de Haas, H. J., Arbustini, E., Fuster, V., Kramer, C. M. \& Narula, J. Molecular imaging of the cardiac extracellular matrix. Circ Res 114, 903-915 (2014).

40. van den Borne, S. W. et al. Molecular imaging of interstitial alterations in remodeling myocardium after myocardial infarction. J Am Coll Cardiol 52, 2017-2028 (2008).

41. Kolodgie, F. D. et al. Targeting of apoptotic macrophages and experimental atheroma with radiolabeled annexin V: a technique with potential for noninvasive imaging of vulnerable plaque. Circulation 108, 3134-3139 (2003).

42. Proost, J. H. \& Meijer, D. K. MW/Pharm, an integrated software package for drug dosage regimen calculation and therapeutic drug monitoring. Comput Biol Med 22, 155-163 (1992).

\section{Acknowledgments}

This research was supported by a research grant from the International Research Fund for Subsidy of Kyusyu University School of Medicine Alumni and the Banyu Fellowship Program sponsored by Banyu Life Science Foundation International to N. Tahara, and research grants from the foundation "De Drie Lichten" and the Dutch Heart Association (Dr. E. Dekker student Grant) to H. de Haas. DTPA-GSAO, DTPA-GSCA, Cy5.5 GSAO were supplied by Covidien Imaging Solutions. We thank H.H. Boersma for performing blood clearance excellent statistical support we thank E. Bagiella, supported by National Institutes of Health Grant UL1TR000067.

\section{Author contributions}

J.N. conceived the study; R.P. developed and produced the targeting agent; M.D. developed the radiolabeling protocols; C.R. conceived the mouse fluorescence experiments; H.Z. conducted the mouse fluorescence experiments; H.Z. and A.P. conducted the mouse imaging experiments; N.T., A.P., T.Y. and T.S. conducted rabbit experiments; J.Z. conducted and analyzed the immunohistochemistry; N.T., H.H., A.P., J.N. performed data analysis; N.T. and H.H. performed statistical analysis; N.T., H.H. and J.N. prepared figures; N.T., H.H. and J.N. wrote the manuscript; J.N. funded the project; H.Z., A.P., R.P., T.Y., J.Z., T.I., R.S., M.D., T.S., A.K., C.R., N.N. and V.F. helped in manuscript writing and editing.

\section{Additional information}

Supplementary Information accompanies this paper at http://www.nature.com/ scientificreports

Competing financial interests: Dr. Dyszlewski is an employee of Covidien Imaging Solutions, Hazelwood, Missouri. Dr. Panduranghi was an employee of Covidien when the project was being conducted. The other authors claim no conflict of interest.

How to cite this article: Tahara, N. et al. Noninvasive Molecular Imaging of Cell Death in Myocardial Infarction using ${ }^{111}$ In-GSAO. Sci. Rep. 4, 6826; DOI:10.1038/srep06826 (2014).

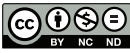

This work is licensed under a Creative Commons Attribution-NonCommercialNoDerivs 4.0 International License. The images or other third party material in this article are included in the article's Creative Commons license, unless indicated otherwise in the credit line; if the material is not included under the Creative Commons license, users will need to obtain permission from the license holder in order to reproduce the material. To view a copy of this license, visit http:// creativecommons.org/licenses/by-nc-nd/4.0/ 